\title{
Bioclimatologie
}

\section{Une méthode de mesure du débit de sève brute dans de petits arbres par bilan de chaleur}

\author{
C. Valancogne et Z. Nasr
}

INRA, Laboratoire de bioclimatologie, centre de Bordeaux, Domaine de la Grande Ferrade, 33140 Pont-de-la-Maye, France

(reçu le 23 janvier 1989, accepté le 28 mars 1989)

\begin{abstract}
Résumé - Le débit de sève brute dans un arbre est calculé à partir du bilan de chaleur d'un élément de volume du tronc entouré par un ruban chauffant délivrant une quantité de chaleur connue. Deux paires de thermocouples mesurent le gradient de température en amont et en aval du volume chauffé. Ils permettent de déterminer les flux de chaleur conductifs dans l'axe du tronc et l'échauffement de la sève. On admet que les pertes de chaleur latérales sont rendues négligeables par l'utilisation d'un manchon fortement isolant. La méthode a été testée d'abord en laboratoire, en conditions stationnaires, en forçant des flux d'eau connus dans des segments de rameaux, puis sous serre avec de petits arbres en pot (de différents diamètres : 1 à $8 \mathrm{~cm}$ ) dont on mesurait la transpiration réelle par pesée. Le débit de sève calculé suit de près la transpiration mesurée. La transpiration journalière est estimée avec une erreur par excès variable, mais qui ne dépasse pas $30 \%$. L'analyse des résultats montre que l'on doit pouvoir améliorer la méthode proposée, d'une part en mesurant les pertes de chaleur latérales, d'autre part en prenant en compte la puissance calorifique stockée dans le volume de tronc considéré.
\end{abstract}

méthode de mesure - débit de sève - arbres - bilan de chaleur

Summary - A heat balance method for measuring the sap flow in small trees. The sap flow rate in trees is estimated from the heat balance computed in a segment of the trunk surrounded with a heating tape delivering a known rate of heat. Two pairs of thermocouples, radially inserted in the upstream and downstream ends of the heated segment of the trunk, are used to determine the conductive heat flows in the axis of the trunk and the heating of the sap (Fig. 1). By using good thermal insulation, it is assumed that the heat flow between the trunk surface and the surrounding air is negligible. The method was tested : a) in the laboratory, by forcing water passage in a branch segment under stationary conditions and by comparing these known water fluxes with the estimated values; $b$ ) in the greenhouse, with small potted trees (1-8 cm diameter), by comparing the actual transpiration rate measured by weighing and the estimated values. The experimental results indicate that the computed sap flow rate closely follows the measured transpiration rate and the daily transpiration flow is evaluated by excess with a variable error not greater than $30 \%$. Analysis of the results shows that this method can be improved by measuring the lateral heat flow and the heat power accumulated in the volume of trunk limited by the heating jacket. An improved sap flow sensor is now being tested.

measurement - sap flow - trees - heat balance

\section{Introduction}

La connaissance des quantités d'eau transpirées par les plantes est importante, tant pour la conduite de l'irrigation des cultures que pour la compréhension du fonctionnement hydrique des plantes.

De nombreuses méthodes permettent d'estimer l'évapotranspiration à des pas de temps variés de l'ordre de l'heure, de la journée ou de la semaine. Chacune a ses avantages et ses limites. Pour les cultures couvrant bien le sol, le choix est large. En revanche, pour les cultures discontinues, ou en rang (vigne, vergers, etc.), on se heurte à des problèmes d'échantillonnage ou de représentativité beaucoup plus délicats quand on calcule le bilan hydrique à partir de mesures d'humidité du sol ou que l'on utilise des lysimètres. Les méthodes micrométéorologiques, de type aérodynamique, telles qu'elles ont été développées avec succès sur couverts continus, ne sont plus directement applicables. De plus, une large fraction du sol est atteinte directement par le rayonnement solaire. Or, les surfaces 
végétales et le sol évaporent différemment. Les méthodes micrométéorologiques basées sur le rapport de Bowen demandent à être appliquées avec prudence, car les surfaces évaporantes ne coïncident pas exactement avec les surfaces échangeant de la chaleur sensible. Enfin, ces méthodes ne permettent pas de distinguer l'évaporation des plantes cultivées de celle du sol ou de la végétation secondaire qui le recouvre. $\mathrm{La}$ mesure du débit de sève brute dans les tiges, les troncs ou les rameaux est, en revanche, un moyen d'évaluer la transpiration (au stockage d'eau près).

Les méthodes à base de traceurs chimiques ou radioactifs ont été essayées, mais sont peu utilisées; elles sont en effet délicates d'emploi et surtout ne permettent guère des observations continues sur de longues périodes.

Les méthodes thermiques sont d'un emploi plus simple. Une source de chaleur est implantée dans le courant de sève brute ou à proximité. Le champ de température au voisinage de cette source est plus ou moins perturbé selon l'importance du flux de sève. L'eau modifie très sensiblement le comportement thermique du matériau qui la contient, en raison de sa forte chaleur spécifique $\left(4180 \mathrm{~J} . \mathrm{kg}^{-1} .{ }^{\circ} \mathrm{C}^{-1}\right.$, contre $1250 \mathrm{~J} . \mathrm{kg}^{-1} .{ }^{\circ} \mathrm{C}^{-1}$ pour le bois sec, ou 800 à $900 \mathrm{~J} . \mathrm{kg}^{-1} .{ }^{\circ} \mathrm{C}^{-1}$ pour des sols secs). Ces méthodes sont maintenant aisées, car on dispose de capteurs de température sensibles, précis et fiables et de systèmes de mesures automatiques informatisés utilisables au champ et de prix raisonnable.

Les méthodes thermiques ont été étudiées depuis de nombreuses années. Les premiers essais ont consisté à suivre la vitesse de déplacement d'une impulsion de chaleur à partir d'une source de chaleur introduite dans le courant de sève. Huber et Schmidt (1937) assimilent la vitesse de déplacement de la sève brute à celle de l'onde de chaleur et appliquent cette méthode à diverses espèces forestières. Marshall (1958), s'inspirant des études théoriques de thermiciens (Carslaw et Jaeger, 1947), établit la relation qui lie en fait la vitesse de la sève à celle de l'onde de chaleur observée. Plusieurs méthodes de mesure de la vitesse de déplacement d'une onde de chaleur ont été ainsi mises en œuvre par divers auteurs (Doley et Grieve, 1966; Cohen et al., 1981, 1985; Closs, 1958; Kunimasa et Kishimoto, 1975; Yamoto et Watanabe, 1980; Cohen, communication personnelle). Ces méthodes sont relativement simples, mais la vitesse réelle de la sève doit être obtenue en appliquant un coefficient de correction empirique à la vitesse mesurée.

Granier $(1985,1987)$ a utilisé pour des arbres une autre méthode thermique. Une aiguille émet- tant une puissance calorifique constante est implantée dans le courant de sève. Une relation est établie entre la température de cette aiguille et la vitesse de la sève qui paraît indépendante de l'espèce. La mesure est très simple, elle réagit très précisément et rapidement aux variations de vitesse de sève; mais le capteur est d'une confection plus délicate.

Toutes ces méthodes ont cependant l'inconvénient majeur de ne fournir que la vitesse moyenne de la sève brute dans les tissus conducteurs actifs. Pour obtenir le débit total de sève, il est donc nécessaire d'estimer l'aire de la section transversale de xylème fonctionnel. Ce n'est aisé que pour des arbres dont le diamètre est assez gros pour permettre un carottage et dont la forme des cernes de bois est régulière. Pour de petits arbres, tels ceux que l'on rencontre dans les vergers modernes, ou pour des plantes annuelles, ce problème n'a guère de solution. Aussi, d'autres méthodes ont-elles été recherchées.

Sakuratani (1981) a eu l'idée de calculer le bilan de chaleur d'un segment de végétal. II l'a appliquée à de petites plantes (tournesol, soja, coton). Nous avons repris le principe de cette méthode et l'avons adapté à la mesure du débit de sève brute dans de petits arbres.

Nous présentons, ci-après, le principe de la méthode de mesure du débit de sève basé sur le bilan de chaleur, les techniques employées et les hypothèses simplificatrices admises au moins dans un premier temps. Des tests ont été effectués, en laboratoire, sur des segments de troncs avec forçage de débits d'eau connus, puis, en serre, sur de jeunes arbres, dont la transpiration fut contrôlée par pesée. Les résultats sont actuellement satisfaisants et devraient pouvoir encore être améliorés en réduisant le nombre des hypothèses simplificatrices admises.

\section{Principe}

Un manchon chauffant (Fig. 1), délivrant une puissance calorifique constante $W$, est disposé autour d'un tronc ou d'une tige. II délimite ainsi un volume $V$, dont il élève la température. II en résulte, au niveau des surfaces externes de cet élément de volume, des flux de chaleur :

- $q_{a m}$ et $q_{a v}$, par conduction selon l'axe du bois, respectivement vers l'amont et vers l'aval;

- $q_{L}$, par conduction/convection vers l'air environnant;

- $q_{c}$, par convection associée au débit de sève $d$.

En raison du principe de conservation de l'énergie, sous réserve qu'il n'y ait pas d'évaporation ou de condensation (chaleur latente), toute 


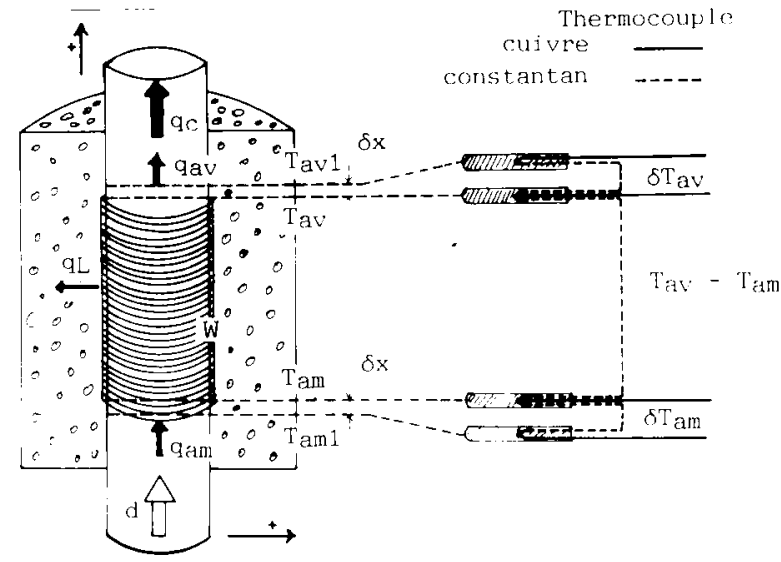

Fig. 1. Schéma du capteur mesurant le débit de sève brute à partir du bilan de chaleur : $W$, puissance calorifique produite par le manchon chauffant (Watt); $q_{a m}$, $q_{a v}$ et $q_{L}$, les flux de chaleur conductifs (Watt); $q_{c}$, le flux de chaleur convectif (Watt) associé au débit de sève $d\left(\mathrm{~m}^{3} . \mathrm{s}^{-1}\right) ; \mathrm{T}_{\mathrm{am}}, \mathrm{T}_{\mathrm{am} 1}, \mathrm{~T}_{\mathrm{av}}$ et $\mathrm{T}_{\mathrm{av1}}$, températures $\left({ }^{\circ} \mathrm{C}\right) ; \delta \mathrm{x}_{\mathrm{am}}$ et $\delta \mathrm{x}_{\mathrm{av}}$, distances entre thermocouples.

la chaleur $W$ fournie à l'élément de volume $V$ doit se retrouver dans ces flux de chaleur et, éventuellement, contribuer à faire varier la température de l'élément de volume.

Le bilan énergétique de l'élément de volume $V$ peut être représenté par l'équation suivante, si l'on considère comme positifs les flux au long de l'axe dirigés vers l'aval et les flux radiaux vers l'extérieur:

$$
\mathrm{W}=\mathrm{q}_{\mathrm{av}}-\mathrm{q}_{\mathrm{am}}+\mathrm{q}_{\mathrm{L}}+\mathrm{q}_{\mathrm{c}}+\mathrm{Q}
$$

avec $Q$, la puissance calorifique mobilisée par le réchauffement de l'élément de volume $\mathrm{V}$.

Le transport de chaleur par convection $q_{c}$, associé au débit de sève, est relié au débit de sève $d$ par la relation suivante :

$$
\mathrm{q}_{\mathrm{c}}=\mathrm{c}_{\mathrm{v}} \mathrm{d}\left(\mathrm{T}_{\mathrm{av}}-\mathrm{T}_{\mathrm{am}}\right)
$$

avec $c_{v}$, la chaleur volumique de la sève brute, qui peut être assimilée à celle de l'eau pure $\left(4,18.10^{6} \mathrm{~J} \cdot \mathrm{m}^{-3} \cdot{ }^{\circ} \mathrm{C}^{-1}\right), \mathrm{T}_{\text {av }}$ et $\mathrm{T}_{\mathrm{am}}$, les températures moyennes des sections transversales limitant le volume $\mathrm{V}$, respectivement à l'aval et à l'amont de l'élément chauffant.

Ainsi, à partir des relations (1) et (2), il est possible de calculer le débit de sève brute, à la condition que $\left(T_{a v}-T_{a m}\right)$ ne soit pas nul :

$$
d=\left(W-q_{a v}+q_{a m}-q_{L}-Q\right) /\left(c_{v}\left(T_{a v}-T_{a m}\right)\right)
$$

En conséquence, pour une puissance calorifique $W$ fournie donnée, la détermination, au même instant, des flux de chaleur $q_{a m}, q_{a v}$, $q_{L}$ et de la puissance calorifique accumulée $Q$ dans le volume $\mathrm{V}$ doit permettre d'estimer le débit de sève brut.

\section{Matériel et Méthode}

\section{Source de chaleur}

Pour les premiers capteurs, la source de chaleur était constituée par un enroulement, autour du tronc, de fil de constantan jointif, alimenté par une tension continue constante, ajustée pour fournir la puissance calorifique voulue. La résistivité électrique du constantan a l'avantage d'être pratiquement indépendante de la température dans nos domaines de travail.

Ce montage de la source chauffante a ensuite été modifié pour s'adapter à la croissance des troncs en diamètre. Le fil de constantan est désormais monté en accordéon sur un ruban adhésif souple que l'on dispose ensuite autour du tronc. Ce système élastique permet ainsi des études de longue durée.

La longueur du manchon chauffant admise est de une à 2 fois le diamètre du tronc.

\section{Détermination des flux de chaleur conductifs axiaux et de l'échauffement de la sève}

D'après la loi de conduction de la chaleur (loi de Fourier), les flux de chaleur par conduction qui traversent les sections transversales à l'amont et à l'aval du volume $V$ sont donnés par les relations :

$$
\begin{aligned}
& q_{a m}=-\alpha A_{a m}(d T / d x)_{a m} \\
& q_{a v}=-\alpha A_{a v}(d T / d x)_{a v}
\end{aligned}
$$

où $\alpha$ est la conductivité thermique du tronc, $A_{a m}$ et $A_{a w}$ respectivement les aires des sections transversales à l'amont et l'aval du volume $\mathrm{V}$, (dT/dx) les gradients de température moyens selon l'axe du tronc, à l'amont et à l'aval.

En pratique, pour évaluer le gradient de température en un point, il faut mesurer l'écart de température $\delta T$ entre 2 points distants de $\delta x$ aussi petit que possible.

Sakuratani, travaillant sur des plantes de faible diamètre (de l'ordre de un à $2 \mathrm{~cm}$ au plus), utilise des thermocouples posés à la périphérie de la tige, ou à peine enfoncés dans la tige, à des distances $\delta \mathrm{x}=1 \mathrm{~mm}$. II considère que ces mesures de températures sont représentatives de la température moyenne des sections transversales.

Notre dispositif étant destiné à des plantes de plus gros diamètre (de petits arbres tels qu'on les rencontre de plus en plus couramment dans les vergers modernes, avec des diamètres de 5 à $10 \mathrm{~cm}$ ), cette hypothèse ne peut plus être admise. Pour estimer la température moyenne de sections transversales de tronc, nous avons alors choisi d'utiliser des sondes de température constituées par des tubes métalliques de $2 \mathrm{~mm}$ de diamètre, contenant un thermocouple cuivreconstantan, emplis de résine époxy pour améliorer le contact thermique entre le thermocouple et le tube. Ces sondes sont insérées dans le tronc après forage d'un trou de $2 \mathrm{~mm}$ de diamètre à l'aide d'une perceuse sur batterie. Un gabarit de guidage est utilisé, lors du forage, pour assurer le meilleur parallélisme possible des sondes et le respect de distances précises entre elles. Deux sondes séparées de $\delta x(2,3$ ou $5 \mathrm{~mm}$ selon les expériences) sont installées à l'amont du manchon chauffant, permettant de mesurer les températures $T_{a m}$ et $T_{a m 1}, 2$ autres de la même manière à l'aval, donnant $T_{a v}$ et $T_{a v 1}$ (Fig. 1). On mesure, en fait, les écarts de température $\delta T_{a m}=T_{a m}-T_{a m 1}, T_{a v}-T_{a m}$, 
$\delta \mathrm{T}_{\mathrm{av}}=\mathrm{T}_{\mathrm{av} 1}-\mathrm{T}_{\mathrm{av}}: \delta \mathrm{T}_{\mathrm{am}} / \delta \mathrm{x}$ et $\delta \mathrm{T}_{\mathrm{av}} / \delta \mathrm{x}$ peuvent être assimilés aux gradients $(d T / d x)_{a m}$ et (dT/dx) $)_{a v}$. Enfin, $T_{a v}-T_{a m}$ donne l'échauffement de la sève lors de son passage dans le manchon chauffant.

\section{Hypothèses simplificatrices}

\section{Hypothèse 1 nul. \\ Le flux de chaleur latéral $q_{L}$ est considéré comme}

L'application de la méthode par Sakuratani (1981) a permis d'évaluer les ordres de grandeur respectifs des différents flux de chaleur (Fig. 2). Ainsi, lorsque le débit de sève croît, la part du flux convectif $q_{c}$ associé au débit de sève croît rapidement et la contribution des flux de chaleur par conduction selon l'axe du tronc, $q_{a m}$ et $q_{a v}$, décroît; $q_{a v}$ devient rapidement négligeable. Dans le dispositif de Sakuratani, qui ne comporte pas d'isolation thermique latérale, le flux de chaleur latéral $q_{L}$, qui représente $50 \%$ de la chaleur $W$ fournie à débit de sève nul, décroît rapidement à moins de $15 \%$. Nous avons donc admis, dans un premier temps, que $q_{L}$ pouvait être considéré comme négligeable en disposant, autour du manchon chauffant, une épaisse couche d'un excellent isolant thermique. Ce choix permet d'éliminer le fluxmètre thermique, dispositif délicat à fabriquer et introuvable dans le commerce pour des flux de chaleur aussi faibles que ceux employés dans la méthode. Après des mousses de polyuréthane en bombe qui se sont révélées souvent peu homogènes et parfois toxiques (à cause des solvants employés), nous avons utilisé comme matériau isolant des plaques souples de mousse de néoprène semblables à celles qu'on utilise pour la confection des combinaisons isothermes de plongée sous-marine.

\section{Hypothèse 2}

La puissance calorifique stockée $Q$ est considérée comme nulle.

Nous admettons, comme Sakuratani et au moins dans un premier temps, que les températures dans le volume se mettent rapidement en équilibre lors de variations du débit de sève, c'est-à-dire que la puissance calorifique $Q$ stockée dans le volume $V$ est pratiquement nulle (régime quasi permanent).

Avec ces 2 hypothèses, l'équation du bilan de chaleur (1) se réduit à :

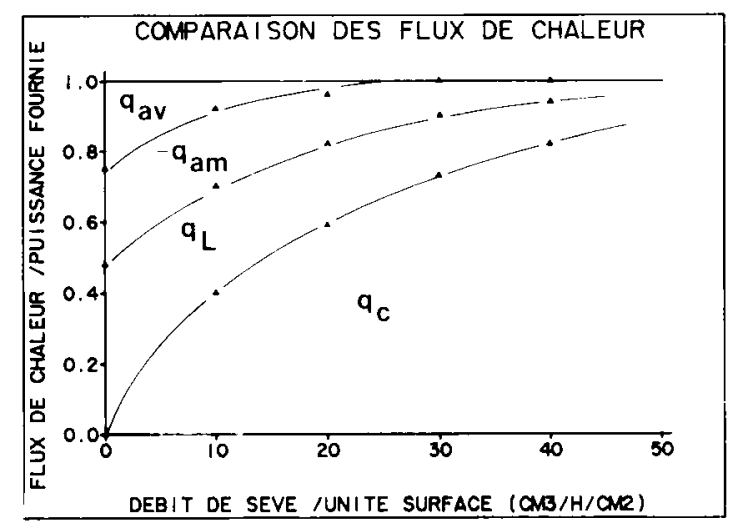

Fig. 2. Evolution des différents termes du bilan de chaleur en fonction du débit de sève rapporté à l'aire de la section transversale de la tige (d'après Sakuratani, 1981).

\section{Hypothèse 3}

Pour déterminer les flux de chaleur par conduction $q_{a m}$ et $q_{a v}$, on admet qu'à débit de sève nul la répartition de la chaleur vers l'amont et vers l'aval est égale.

Pour évaluer les flux de chaleur par conduction selon l'axe du tronc, $q_{a m}$ et $q_{a v}$, il faut connaitre la conductivité thermique $\alpha$ du tronc, les aires des sections transversales amont et aval $A_{a m}$ et $A_{a v}$.

Pour le tournesol et le soja, Sakuratani a évalué $\alpha$ à respectivement 0,59 et $0,50 \mathrm{~W} \cdot \mathrm{m}^{-1} \cdot{ }^{\circ} \mathrm{C}-1$. En fait, pour des arbres, $\alpha$ peut varier dans une gamme assez étendue, de l'ordre de 0,35 à $0,65 \mathrm{~W} \cdot \mathrm{m}^{-1} .{ }^{\circ} \mathrm{C}^{-1}$ (d'après Marshall, 1958; Cohen et al., 1981, 1985). La mesure de $\alpha$ est en fait délicate et complexe.

Par ailleurs, l'estimation précise des aires $A_{a m}$ et $A_{a v}$ est souvent difficile. La forme des troncs n'est en général pas parfaitement circulaire par suite de l'existence de contraintes mécaniques non isotropes (cf. vents dominants). Enfin, la distance $\delta x$ entre les sondes de température à l'amont ou à l'aval du manchon chauffant n'est pas aussi bien définie qu'on ne le voudrait; les forets utilisés pour la mise en place des sondes, étant de petit diamètre $(2 \mathrm{~mm})$, ont tendance à se déformer en fonction de la variation des contraintes rencontrées au cours de la pénétration à travers les fibres du bois.

On peut regrouper ces différents paramètres en un coefficient unique de conduction qui sera différent à l'amont et à l'aval, principalement en raison des valeurs de $\delta x$ dissemblables:

$$
\begin{gathered}
\mathrm{K}_{\mathrm{am}}=\alpha \mathrm{A}_{\mathrm{am}} / \delta \mathrm{x}_{\mathrm{am}} \\
\mathrm{K}_{\mathrm{av}}=\alpha \mathrm{A}_{\mathrm{av}} / \delta \mathrm{x}_{\mathrm{av}}
\end{gathered}
$$

Pour déterminer ces coefficients de conduction (exprimés en $\mathrm{W} .{ }^{\circ} \mathrm{C}-1$ ), nous admettrons que la chaleur fournie par le manchon chauffant, à débit de sève nul, se répartit également vers l'amont et vers l'aval. Cette hypothèse n'est acceptable que si le capteur est installé au niveau d'une zone homogène et régulière du tronc (absence de départ de branches ou de blessures).

Compte tenu des hypothèses 1 et 2 , on a donc les relations suivantes:

$$
\begin{aligned}
& W / 2=q_{a v}=-K_{a v} / \delta T_{a v o} \\
& W / 2=-q_{a m}=K_{a m} / \delta T_{a m 0}
\end{aligned}
$$

$K_{a m}$ et $K_{a v}$ peuvent donc être déterminés à partir de valeurs de $\delta T_{a m}$ et $\delta T_{a v}$ à débit de sève nul, soit respectivement $\delta \mathrm{T}_{\text {amo }}$ et $\delta \mathrm{T}_{\text {avo. }}$ Le repérage des conditions de débit de sève nul est relativement aisé. C'est à débit de sève nul que les flux conductifs selon l'axe du tronc sont maximaux en valeur absolue. C'est donc à cette condition que les valeurs absolues de $\delta T_{\text {am }}$ et $\delta \mathrm{T}_{\mathrm{av}}$ sont maximales, toutes autres conditions étant égales. Notons que pour un même débit de sève, en cas de sécheresse prolongée, les coefficients de conduction $\mathrm{K}_{\mathrm{am}}$ et $\mathrm{K}_{\mathrm{av}}$ diminuent avec le dessèchement du bois, entraînant une augmentation des $\delta T_{a m}$ et $\delta T_{a v}$. Pour évaluer les coefficients de conduction $K_{a m}$ et $K_{a v}$, il suffit donc de relever les plus fortes valeurs absolues de $\delta \mathrm{T}_{\mathrm{am}}$ et $\delta \mathrm{T}_{\mathrm{av}}$, à condition toutefois que les plantes ne subissent pas de stress hydrique en permanence. Ces conditions ont le plus de chance d'être rencontrées vers la fin de la nuit, après des précipitations ou un arrosage.

Avec cette hypothèse de répartition égale de la chaleur fournie vers l'amont et l'aval, à débit de sève nul, il est possible d'évaluer les coefficients de conduc- 
tion sans étalonnage préalable, les capteurs étant en place. Le débit de sève peut être alors calculé à tout moment à partir de 3 mesures $\left(\delta \mathrm{T}_{\mathrm{am}}, \delta \mathrm{T}_{\mathrm{av}}, \mathrm{T}_{\mathrm{av}}-\mathrm{T}_{\mathrm{am}}\right)$ selon la relation issue de (6) :

$$
\mathrm{d}=\left(\mathrm{W}-\mathrm{K}_{\mathrm{am}} \delta \mathrm{T}_{\mathrm{am}}+\mathrm{K}_{\mathrm{av}} \delta \mathrm{T}_{\mathrm{av}}\right) /\left(\mathrm{C}_{\mathrm{v}}\left(\mathrm{T}_{\mathrm{av}}-\mathrm{T}_{\mathrm{am}}\right)\right)
$$

\section{Dispositifs expérimentaux}

Tests en régime quasi permanent avec forçage de débits d'eau connus

Un segment de rameau d'arbre, équipé d'un capteur de débit de sève, est raccordé à un réservoir hydropneumatique. Différents débits d'eau sont obtenus en ajustant la pression d'air dans le réservoir. L'eau sortant du segment de rameau est recueillie dans un flacon. La variation de poids du flacon, suivie continuellement à l'aide d'une balance électronique associée à un micro-ordinateur, permet de calculer le débit d'eau.

Les coefficients de conduction $K_{a m}$ et $K_{a v}$ sont déterminés à débit d'eau nul. Les valeurs de $\delta T_{a m}$, $T_{a v}-T_{a m}, \delta T_{a v}$ ont été notées pour différents débits d'eau, une fois le régime thermique stationnaire atteint.

Tests en conditions réelles avec contrôle de la transpiration par pesée

Des arbres plantés dans des pots enveloppés de film en PVC sont placés sur une balance électronique reliée à un micro-ordinateur. Les variations de poids à des intervalles de temps précis sont ainsi enregistrées pour calculer la transpiration. Ces expériences sont faites en serre plutôt qu'au champ, pour éviter les effets perturbateurs du vent sur les pesées. Le capteur de débit de sève est installé vers la partie inférieure du tronc dans une zone de forme régulière.

Les coefficients de conduction $K_{a m}$ et $K_{a v}$ sont déterminés à partir des maxima des valeurs absolues de $\delta \mathrm{T}_{\mathrm{am}}$ et $\delta \mathrm{T}_{\mathrm{av}}$ observés sur plusieurs journées.

\section{Résultats}

Les Figures $3 a$ et $b$ montrent les résultats obtenus lors des tests en régime quasi permanent avec forçage de débits d'eau. Le débit d'eau calculé est toujours très proche du débit d'eau mesuré. Si l'on prend en compte l'ensemble des expériences faites dans ces conditions avec différents rameaux, on constate une tendance presque systématique de la méthode à surestimer le débit d'eau.

Les Figures $4 a, b$ et $c$ montrent pour 3 arbres de différents diamètres, placés en conditions réelles, l'évolution, au cours de 2 journées consécutives, du débit de sève calculé par rapport à la transpiration mesurée. Sur la Figure $4 b$, on a en outre porté le rayonnement global transmis par la serre. Le débit de sève calculé est très voisin de la transpiration mesurée. Pour les plus gros troncs (ex.: Fig. 4c), il faut noter un retard notable de l'évolution du débit de sève calculé par rapport à la transpiration mesurée (de l'ordre de $1 \mathrm{~h}$ à 1 h30 dans le cas présenté).
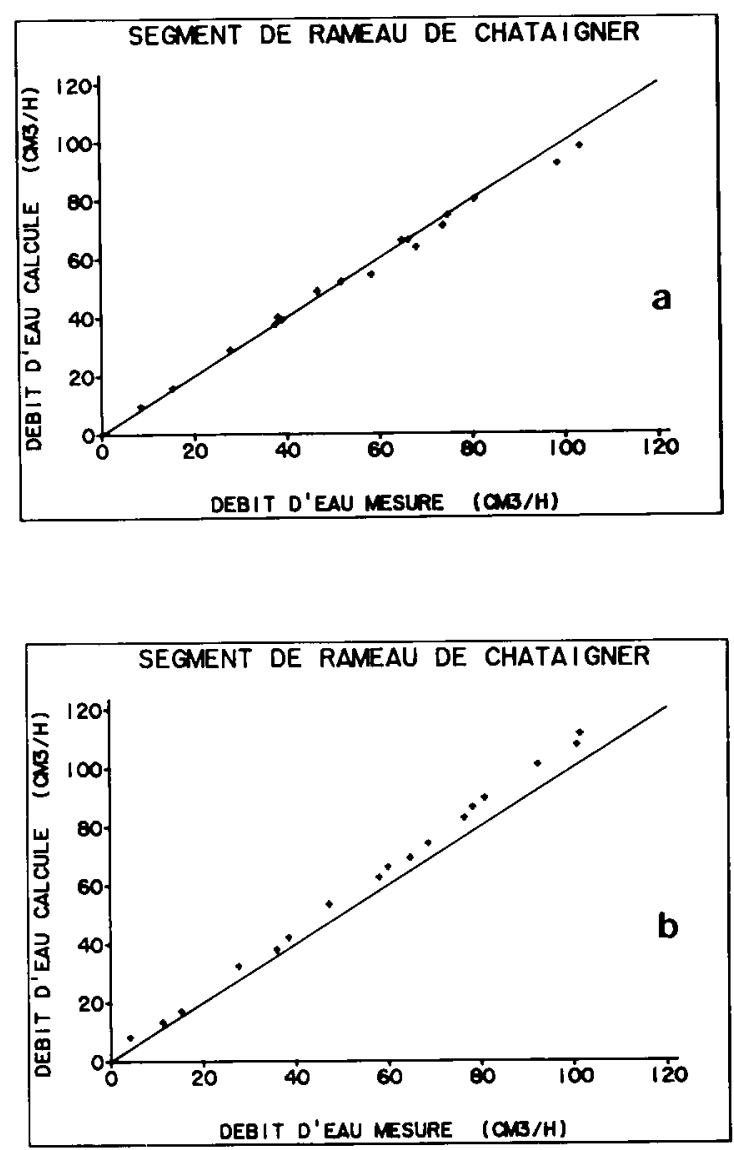

Fig. 3. Comparaison entre le débit d'eau calculé et le débit d'eau mesuré dans des segments de rameau de chataîgner : a. Diamètre du tronc $=1,1 \mathrm{~cm}$, longueur du manchon chauffant $=2 \mathrm{~cm}, \delta x=0,3 \mathrm{~cm}$, puissance de l'élément chauffant $W$ $=0,112 \mathrm{~W}$. b. Diamètre $=1,3 \mathrm{~cm}$, longueur du manchon chauffant $=1,5 \mathrm{~cm}, \delta x=0,3 \mathrm{~cm}, W=0,122 \mathrm{~W}$. Dans les 2 cas, le coefficient de corrélation est meilleur que 0,99; la droite de pente 1 est présentée comme référence.

La relation, pour ces même arbres, entre débit de sève calculé et transpiration mesurée est présentée dans les Figures $5 a$ et $b$. Les Figs $6 a, b$ et $\mathrm{c}$ montrent, pour les 3 arbres de la Fig. 4, l'évolution sur $24 \mathrm{~h}$ du débit de sève calculé chaque heure, en fonction de la transpiration mesuree. On note un effet d'hystérésis d'autant plus marqué que le tronc est plus gros.

A l'échelle journalière, le débit de sève calculé est souvent supérieur à la transpiration mesurée, sans excéder $30 \%$ de celle-ci (Fig. $7 a$ et b). Les valeurs de débit de sève calculé inférieures à la transpiration mesurée correspondent le plus souvent à des périodes de déficit hydrique prononcé. II se peut que, dans ces conditions, les réserves en eau des arbres diminuent et ne se reconstituent pas la nuit.

\section{Discussion}

En l'absence de tout étalonnage préalable, la méthode du bilan de chaleur que nous propo- 

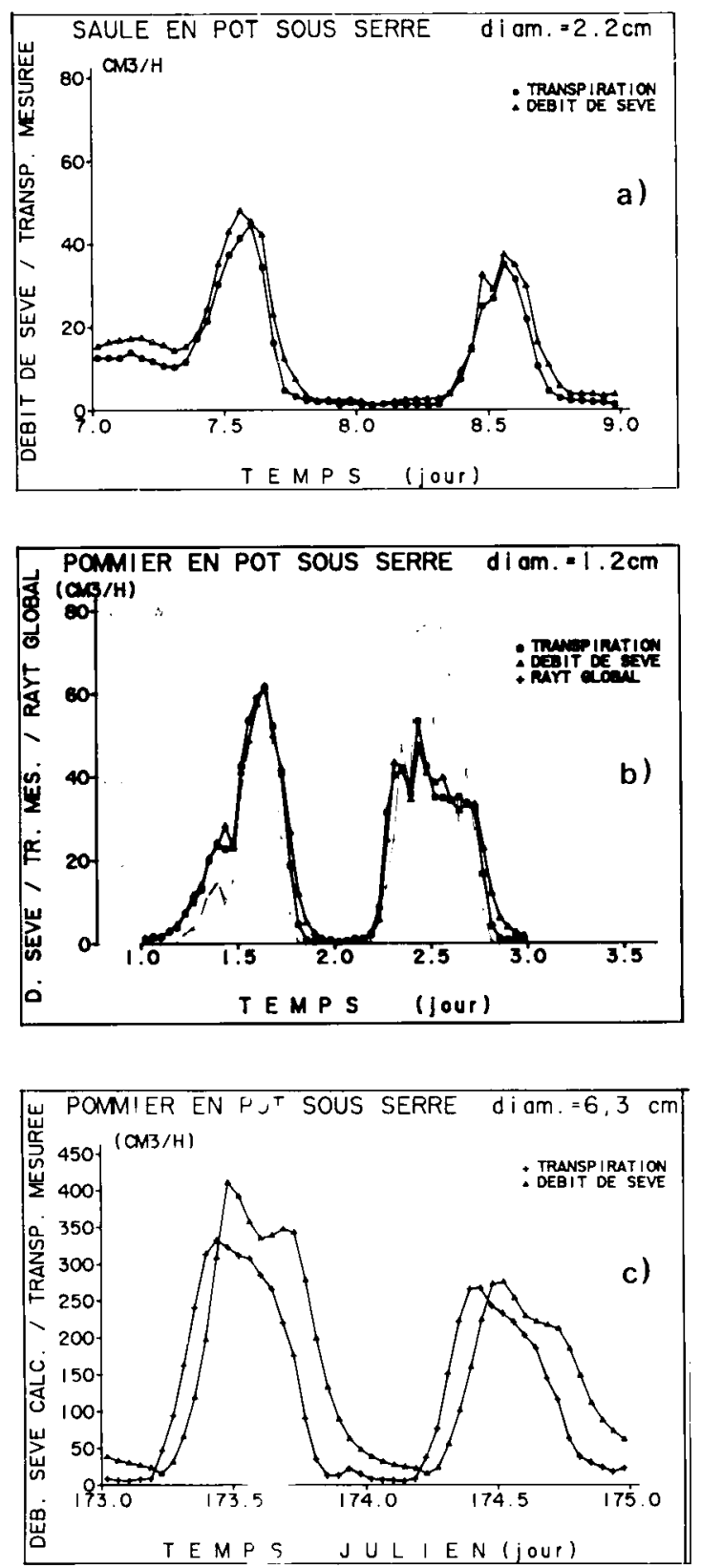

Fig. 4. Evolution du débit de sève calculé et de la transpiration mesurée au cours de 2 journées consécutives pour 3 arbres cultivés en pot sous serre. a. Diamètre $=2,2 \mathrm{~cm}$, longueur du manchon chauffant $=3 \mathrm{~cm}, \delta x=0,3 \mathrm{~cm}$, $W=0,132 \mathrm{~W}$. $\mathrm{b}$. Diamètre $=1,2 \mathrm{~cm}$, longueur du manchon chauffant $=2 \mathrm{~cm}, \delta x=0,4 \mathrm{~cm}, \mathrm{~W}=0,129 \mathrm{~W}$. c. Diamètre $=$ $6,3 \mathrm{~cm}$, longueur du manchon chauffant $=8 \mathrm{~cm}, \delta x=0,5 \mathrm{~cm}$, $W=0,939 \mathrm{~W}$.

sons pour mesurer le débit de sève donne des résultats relativement satisfaisants, mais insuffisants pour un certain nombre d'études fines concernant le fonctionnement hydrique des cultures.

Nous allons discuter les hypothèses que nous avons admises et examiner divers points de la méthode qui peuvent être critiqués.
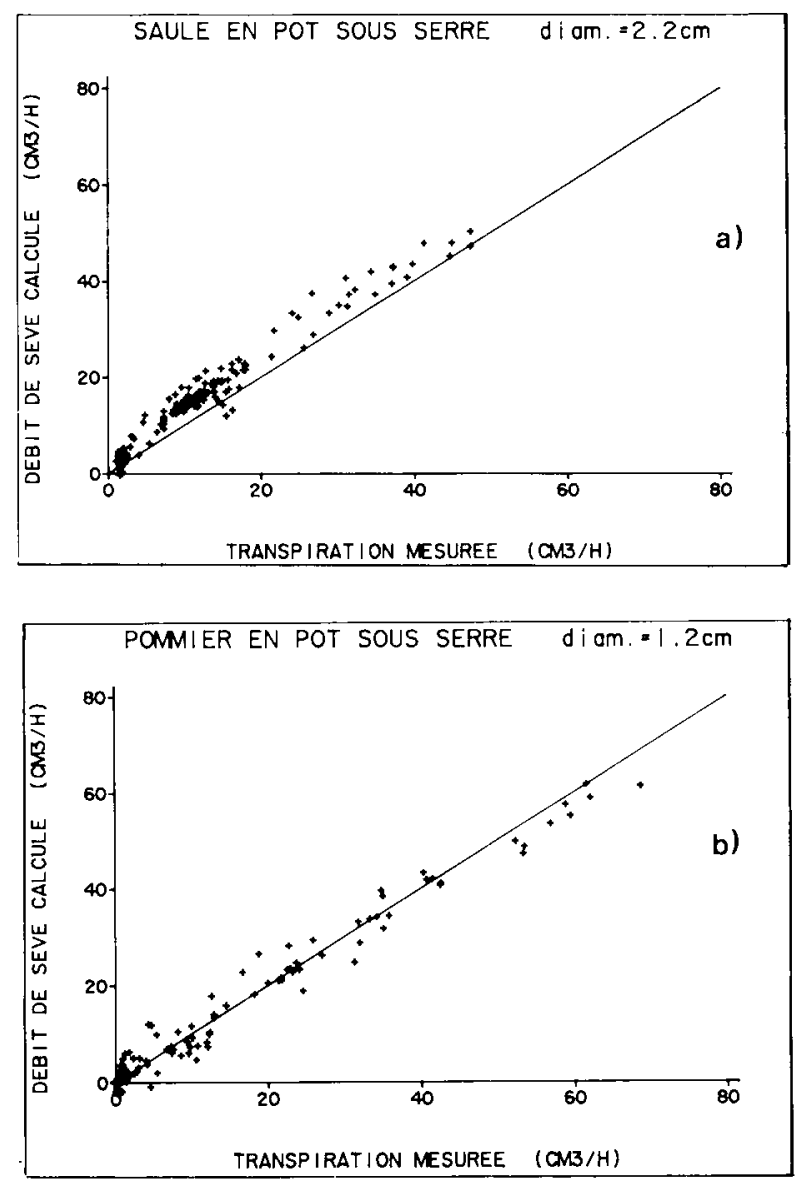

Fig. 5. Relation entre le flux de sève calculé et la transpiration mesurée à l'échelle horaire pour les arbres présentés à la Fig. $4 a$ et $b$.

\section{Effet de l'hypothèse de la nullité des pertes de chaleur latérale $q_{L}$}

Si on examine les coefficients de conduction $\mathrm{K}_{\mathrm{am}}$ et $K_{a v}$ estimés, les capteurs étant en place, on constate qu'ils sont systématiquement supérieurs de 15 à $100 \%$ aux coefficients attendus, compte tenu des ordres de grandeur de la conductivité thermique $\alpha$ de bois jeunes $(0,50$ à 0,60 W. $\left.\mathrm{m}^{-1} \cdot{ }^{\circ} \mathrm{C}^{-1}\right)$, des aires des sections transversales $A$ et des distances $\delta x$ entre les sondes de température situées à l'amont et à l'aval du manchon chauffant $(k=\alpha A / \delta x)$.

Or, il est quasiment improbable que les erreurs sur $A$ et $\delta x$ soient toujours dans le même sens pour toutes les expériences réalisées. Une erreur systématique peut donc être envisagée.

A débit de sève nul, l'équation (1) du bilan de chaleur s'écrit strictement :

$$
W=q_{a v 0}-q_{a m 0}+q_{L 0}+Q
$$

Elle se réduit encore, si l'on admet $Q \approx 0$, hypothèse qui paraît raisonnable lorsque la condition de débit de sève nul est réalisée, c'est- 

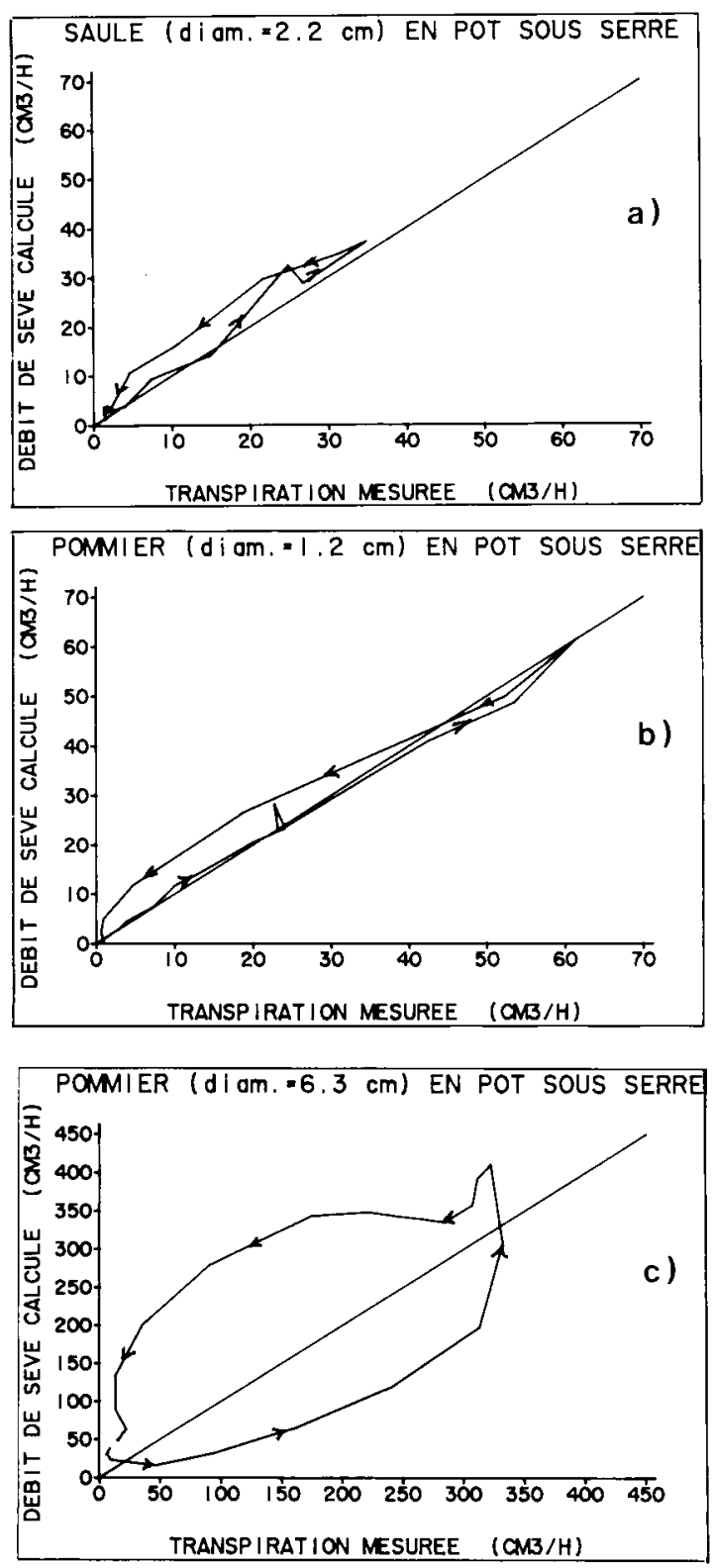

Fig. 6. Déphasage observé au cours d'une journée dans l'évolution respective du flux de sève calculé et de la transpiration mesurée à l'échelle horaire pour les 3 arbres de la Fig. 4.

à-dire au cours de la nuit. D'où, en tenant compte des valeurs de $q_{a m 0}$ et $q_{a v 0}$ attendues en fonction de $\delta \mathrm{T}_{\mathrm{amo}}$ et $\delta \mathrm{T}_{\mathrm{avo}}$ :

$$
W \approx k\left(\delta T_{a m 0}-\delta T_{a v 0}\right)+q_{L o}
$$

Les pertes latérales à débit de sève nul peuvent donc être estimées à :

$$
\mathrm{q}_{\mathrm{L} 0} \approx \mathrm{W}-\alpha \mathrm{A}\left(\delta \mathrm{T}_{\mathrm{amo}}-\delta \mathrm{T}_{\mathrm{avo}}\right) / \delta \mathrm{x}
$$

Ainsi, pour les différentes expériences réalisées en laboratoire et en serre, les pertes latérales représentent 15 à $50 \%$ de la puissance calorifique $\mathrm{W}$ fournie. L'isolation thermique renforcée réduit généralement l'importance des pertes de chaleur latérales, mais elle est très loin de les supprimer.
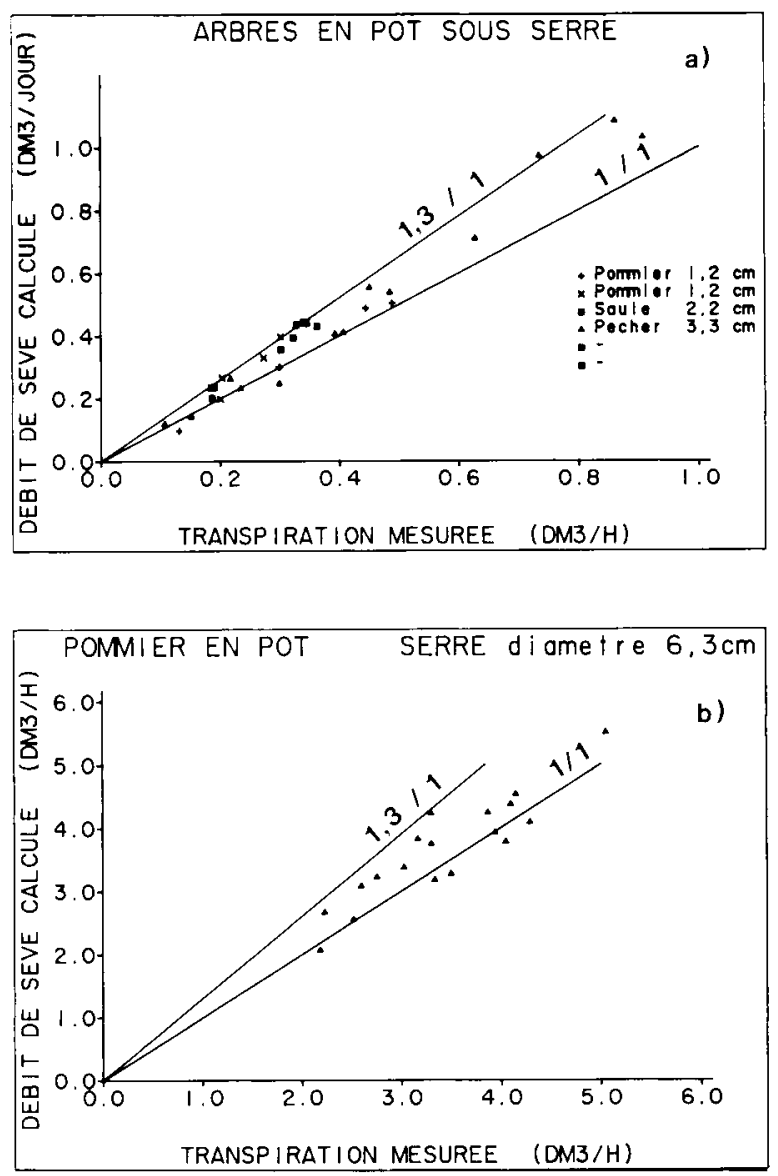

Fig. 7. Relation entre débit de sève calculé et transpiration mesurée, à l'échelle journalière, pour des arbres de différents diamètres. La droite inférieure a une pente égale à 1, la droite supérieure correspond à une surestimation de la transpiration de $30 \%$ (pente 1,30 ).

La prise en compte de ces pertes à l'aide d'un fluxmètre thermique doit améliorer la réponse du capteur.

Le fait d'avoir négligé les pertes de chaleur latérales peut expliquer, au moins pour une part, la surestimation assez systématique du débit de sève. Le débit de sève réel $d_{r}$ est donné en toute rigueur par l'équation (3) :

$$
\left.d_{r}=\left(W-q_{a v}+q_{a m}-q_{L}-Q\right) /\left(T_{a v}-T_{a m}\right)\right)(15)
$$

Nous admettrons, pour simplifier l'analyse, que la puissance calorifique $Q$ accumulée dans le volume délimité par le manchon chauffant est nulle (régime quasi permanent).

Soit $k_{a m}$ et $k_{a v}$, les coefficients de conduction réels. Ils peuvent être estimés de la même manière que $\mathrm{K}_{\mathrm{am}}$ et $\mathrm{K}_{\mathrm{av}}$ à partir de $\delta \mathrm{T}_{\mathrm{am}}$ et $\delta \mathrm{T}_{\mathrm{av}}$ à débit de sève nul, en prenant en compte les pertes de chaleur latérales observées dans ces conditions :

$$
k_{a m}=\left(W-q_{L O}\right) /\left(2 \delta T_{a m 0}\right)
$$

et

$$
k_{a v}=-\left(W-q_{L 0}\right) /\left(2 \delta T_{a v 0}\right)
$$


d'où :

$$
\begin{aligned}
& \mathrm{K}_{\mathrm{am}}=\mathrm{K}_{\mathrm{am}}-\mathrm{q}_{\mathrm{Lo}} /\left(2 \delta \mathrm{T}_{\mathrm{amo}}\right) \\
& \mathrm{K}_{\mathrm{av}}=\mathrm{K}_{\mathrm{av}}+\mathrm{q}_{\mathrm{LO}} /\left(2 \delta \mathrm{T}_{\mathrm{avo}}\right)
\end{aligned}
$$

L'équation (15) clonnant le débit de sève réel devient donc :

$d_{r}=\left(W-q_{L}+k_{a v} \cdot \delta T_{a v}-k_{a m} \cdot \delta T_{a m}\right) /\left(c_{v}\left(T_{a v}-T_{a m}\right)\right)$

d'où, en se basant sur les relations (11), (16) et (17) :

$$
\begin{aligned}
\mathrm{d}_{\mathrm{r}}=\mathrm{d} & -\mathrm{q}_{\mathrm{L}}\left(1-\mathrm{q}_{\mathrm{LO}} / \mathrm{q}_{\mathrm{L}}\left(\delta \mathrm{T}_{\mathrm{av}} / 2 \delta \mathrm{T}_{\mathrm{avo}}\right.\right. \\
& \left.+\delta \mathrm{T}_{\mathrm{am}} / 2 \delta \mathrm{T}_{\mathrm{amo} 0}\right) /\left(\mathrm{c}_{\mathrm{v}}\left(\mathrm{T}_{\mathrm{av}}-\mathrm{T}_{\mathrm{am}}\right)\right)
\end{aligned}
$$

Lorsque le débit de sève augmente, $\mathrm{q}_{\mathrm{LO}} / \mathrm{q}_{\mathrm{L}}$ croît alors que le terme $\left(\delta \mathrm{T}_{\mathrm{av}} / 2 \delta \mathrm{T}_{\mathrm{av0}}+\delta \mathrm{T}_{\mathrm{am}} /\right.$ $2 \delta \mathrm{T}_{\mathrm{amo}}$ ) décroît rapidement. Le sens de l'erreur sur le débit de sève calculé dépend de la manière dont ces 2 termes varient avec le débit de sève. Si l'on se base sur les résultats publiés par Sakuratani (1981) (Fig. 2), le terme $\mathrm{q}_{\mathrm{LO}} / \mathrm{q}_{\mathrm{L}}$ croît moins vite que le terme $\left(\delta \mathrm{T}_{\mathrm{av}} / 2 \delta \mathrm{T}_{\mathrm{av} 0}+\right.$ $\delta \mathrm{T}_{\mathrm{am}} / 2 \delta \mathrm{T}_{\mathrm{amo}}$ ) ne décroît lorsque le débit réel augmente. Le produit de ces 2 termes décroît à partir de 1 pour se rapprocher de plus en plus lentement de 0 . On a bien alors une erreur systématique par excès sur le débit de sève calculé par notre méthode, qui ne tient pas compte des pertes de chaleur latérales à partir du capteur.

Dans la réalité, le problème des pertes de chaleur latérales est complexe. II dépend, en effet, non seulement du débit de sève mais aussi des variations de température dans l'environnement.

\section{Echauffement du tronc. Possibilité d'erreurs dues à la puissance calorifique stockée}

En l'absence de débit de sève, la chaleur ne peut s'évacuer que par conduction le long du bois ou latéralement à travers l'isolant. La température dans le volume délimité par le manchon chauffant est donc plus élevée lorsque le débit de sève est nul (Fig. 8). Plus le débit de sève est élevé, plus grande est la quantité de chaleur évacuée par celle-ci, donc plus l'écart entre la température de cette zone et celle de l'air sera réduit.

Pour avoir une précision suffisante sur les températures, on est pratiquement amené à tolérer des écarts de température par rapport à l'air de l'ordre de 12 à $15^{\circ} \mathrm{C}$ à débit de sève nul. Lorsque le débit de sève est fort, en milieu de journée par exemple, ces écarts deviennent faibles, de l'ordre de $1^{\circ} \mathrm{C}$.

Le matin, quand la sève se met en mouvement, le volume $V$ du tronc délimité par le manchon chauffant est très chaud, puis la température chute par suite du mouvement de sève, la sève arrivant étant plus froide (à une température voisine de celle de l'air). La puissance calorifique

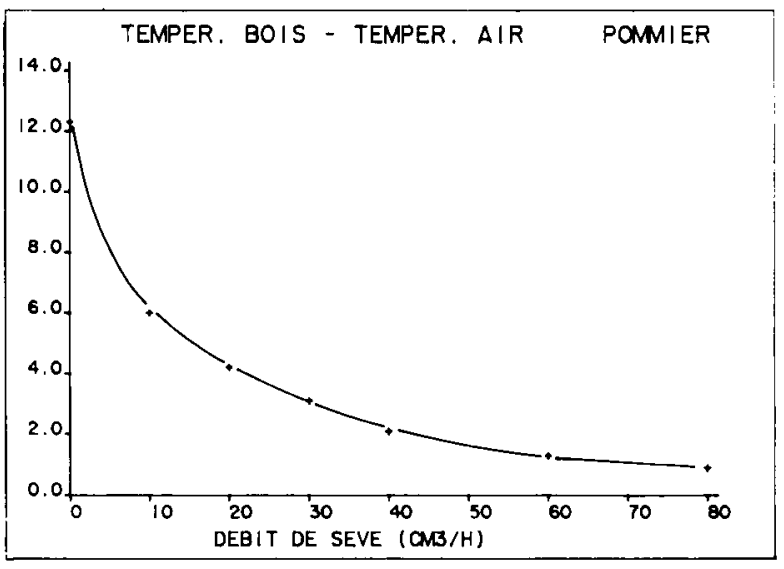

Fig. 8. Variation de la température au milieu du volume délimité par le manchon chauffant en fonction du débit de sève, pour un petit pommier (diamètre de tronc $=1,4 \mathrm{~cm}$ ).

Q ainsi libérée s'ajoute à celle qui est fournie par l'élément chauffant. Si l'on ne tient pas compte de cet apport de chaleur, on sous-estime donc le débit de sève. L'inverse se produit la nuit, à l'occasion du réchauffement du volume $V$. Le déphasage entre débit de sève calculé et transpiration mesurée rencontré dans les expériences de serre (Fig. $6 a, b$ et $c$ ) peut s'expliquer en partie par ce phénomène. Ce problème, peu important sur de petits troncs, le devient pour de plus gros troncs, où le bois de cœur, inactif vis-à-vis de la sève, occupe une surface croissante. $\mathrm{Si}$ l'on considère le débit de sève journalier, ce type d'erreur est moins notable, car il y a une certaine compensation au cours de la journée.

\section{Estimation de la puissance de l'élément chauffant nécessaire}

La puissance calorifique $W$ fournie par l'élément chauffant, à débit de sève nul, se répartit également le long de l'axe du bois vers l'amont et l'aval $\left(q_{a m 0}=q_{a v o}\right)$; en outre, une part s'évacue latéralement $\left(q_{\mathrm{LO}}\right)$. L'équation du bilan énergétique s'écrit alors :

$$
\mathrm{W}=\mathrm{q}_{\mathrm{av0}}-\mathrm{q}_{\mathrm{am} 0}+\mathrm{q}_{\mathrm{L} 0}=2 \mathrm{q}_{\mathrm{av} 0}+\mathrm{q}_{\mathrm{LO}}
$$

Si l'on se base sur les estimations des pertes latérales $q_{L_{0}}$ observées dans nos expériences, on peut considérer que :

$$
q_{L, 0} \approx 1 / 3 W
$$

En conséquence, l'ordre de grandeur des flux de chaleur conductifs au long de l'axe du bois, à débit de sève nul, est :

$$
q_{a v 0}=-q_{a m 0} \approx 1 / 3 \mathrm{~W}
$$

Nos différentes expériences ont aussi montré que, pour avoir une précision raisonnable sur les mesures des flux conductifs, il faut que les écarts de température à débit de sève nul, $\delta T_{a m o}$ et 
$\delta T_{\text {avo }}$ soient de l'ordre de $1^{\circ} \mathrm{C}$ pour $\delta x=0,5 \mathrm{~cm}$. $\mathrm{Si}$ l'on considère que la conductivité thermique du bois $\alpha$ est de l'ordre de $0,6 \mathrm{~W} \cdot \mathrm{m}^{-2}{ }^{\circ}{ }^{\circ} \mathrm{C}-1$, on peut ainsi estimer la puissance $W$ (en Watt) à fournir par l'élément chauffant :

$$
\mathrm{W} \approx 3 \alpha \mathrm{A} \delta \mathrm{T} / \delta \mathrm{x} \approx 3,6 \mathrm{~A}
$$

avec $A$, l'aire de la section transversale du tronc au niveau du capteur de débit de sève (en $\mathrm{m}^{2}$ ).

Un ajustage de la tension d'alimentation du capteur peut être réalisé ensuite pour éviter un échauffement excessif du bois au niveau du capteur lorsque le débit de sève est nul.

\section{Conclusion}

L'intérêt de la méthode du bilan de chaleur pour évaluer le débit de sève d'arbres, au moins de petite taille, est confirmé.

Malgré une approche simplifiée limitée à la mesure de 3 écarts de température, et en l'absence d'étalonnage, le débit de sève peut être estimé avec une erreur par excès qui varie, mais ne dépasse pas $30 \%$ dans le cadre de nos essais. Le débit de sève ainsi calculé suit de près les variations de transpiration au cours de la journée. En l'état, la méthode proposée peut suffire pour un certain nombre d'applications pratiques ne nécessitant pas une grande précision : contrôle de l'irrigation, repérage de stress hydrique durable, etc.

La méthode a été appliquée à des rameaux ou à des troncs mesurant jusqu'à $8 \mathrm{~cm}$ de diamètre. Par son principe, elle est aussi applicable à des racines. Rien n'interdit de penser qu'elle puisse être utilisée pour des diamètres supérieurs; la puissance de l'élément chauffant doit être accrue en proportion de l'augmentation de l'aire de la section transversale au niveau de la zone de mesure.

Pour des études nécessitant des évaluations plus précises du débit de sève, nous proposons en premier lieu de mesurer les pertes de chaleur latérales du capteur. Nos essais montrent qu'elles ne peuvent être réduites de façon significative avec de bons isolants classiques du commerce. L'utilisation d'un fluxmètre thermique, comme Sakuratani, est donc inévitable. La réalisation en est certes difficile, mais tout à fait concevable; elle est maintenant effectuée dans notre laboratoire.
Pour des arbres, où le volume délimité par le capteur de débit de sève est plus important que dans le cas des travaux de Sakuratani, il est probablement nécessaire d'estimer la puissance calorifique stockée, qui peut prendre une certaine importance à certaines heures de la journée. Négliger ce terme peut conduire à une sous-estimation du débit de sève en début de journée et à une surestimation dans la soirée.

Un nouveau capteur prenant en compte ces remarques est actuellement à l'étude. Plus perfectionné (2 mesures de température supplémentaires sont nécessaires), il tient compte de l'analyse effectuée ci-dessus et doit permettre d'améliorer sensiblement la précision.

\section{Références}

Carslaw H.S. \& Jaeger J.C. (1947) Conduction of Heat in Solids. Oxford University Press, London. Nouvelle édition révisée, Clarendon Press, Oxford, 1986

Closs R.L. (1958) The heat pulse method for measuring rate of sap flow in a plant stem. N. Z. Sci. Rev. 1, 281-288

Cohen Y., Fuchs M. \& Green G.C. (1981) Improvment of heat pulse method for determining sap flow in trees. Plant Cell Environ. 4, 391-397

Cohen Y., Kelliher F.M. \& Black T.A. (1985) Determination of sap flow in Douglas-fir trees using the heat pulse technique. Can. J. For. Res. 15, 422-428

Doley D. \& Grieve B.J. (1966) Measurement of sap flow in a eucalypt by thermoelectric methods. Aust. For. Res. 2, 3-27

Granier A. (1985) Une nouvelle méthode pour la mesure du flux de sève brute dans le tronc des arbres. Ann. Sci. For. 42, 193-200

Granier A. (1987) Mesure du flux de sève brute dans le tronc du Douglas par une nouvelle méthode thermique. Ann. Sci. For. 44, 1-14

Huber B. \& Schmidt E. (1937) Eine Kompensationsmethode zur thermoelktrischen Messung langsamer Saftströme. Ber. Dtsch. Bot. Ges. 55, 514-529

Kunimasa K. \& Kishimoto J. (1975) Measurement of sap flow by heat pulse method (texte en japonais, résumé en japonais, résumé et légendes en anglais). Bull. Tottory Forest Univ. (Japon) 8, 101-107

Marshall D.C. (1958) Measurement of sap flow in conifers by heat pulse transport. Plant Physiol. (Bethesda) 33, 385-396

Sakuratani T. (1981) A heat balance method for measuring water flow in the stem of intact plant. J. Agric. Meteorol. 37, 9-17

Yamamoto T. \& Watanabe S. (1980) Measurements of sap velocities in stems, peduncles and petioles of Pear trees by a heat pulse method (texte en japonais, résumé, formules et légendes en anglais). J. Jpn Soc. Hortic. Sci. 49, 311-325 